\title{
Odontogenic Myxoma
}

\author{
Naresh Kumar · Munish Kohli · Saumya Pandey • \\ Poonam Agarwal
}

Received: 29 January 2010/ Accepted: 12 October 2010/Published online: 9 December 2010

(C) Association of Oral and Maxillofacial Surgeons of India 2010

\begin{abstract}
Odontogenic myxoma is a rare, benign, locally aggressive and non metastasizing neoplasm which is believed to arise from the odontogenic ectomesenchyme and bears a close microscopic resemblance to mesenchymal portion of a tooth germ. This is a case report of odontogenic myxoma in a 32 year old female patient and the treatment rendered to her.
\end{abstract}

\section{Keyword Odontogenic myxoma}

\section{Introduction}

Odontogenic myxoma is a rare benign tumour of the jaw and characteristically presents as a slow, painless, bony expansion with resultant facial deformity. It is most commonly associated with an unerupted tooth and probably arises from the mesenchymal portion of the tooth germ [1].

According to a study by Regezi et al. [2], it comprised $3 \%$ of the 706 odontogenic tumors studied by Daley et al. [3] found them to comprise $5.1 \%$ of the 445 odontogenic tumors of their study. It usually presents itself in the second to the third decade of life [4], although some authors have reported the age of occurrence as 15-65 years yet few cases [5] have been reported in pediatric age group in a 17-month old baby.

There is no sex predilection [4], but according to some authors there is slight female predilection and male to female ratio is $1: 1.5[2,8]$. The tumour may be found in almost any area of the jaws [4] and mandible is more commonly affected than maxilla (3:1) [9].

N. Kumar $(\bowtie) \cdot$ M. Kohli · S. Pandey · P. Agarwal Saraswati Dental College and Hospital, Lucknow, India e-mail: drnsharma2001@gmail.com
This is a case report of a swelling of mandible in a 32-years old female, histopathologically diagnosed as odontogenic myxoma.

\section{Case Report}

A 32-year old female patient presented to the department of Oral and Maxillofacial Surgery, SDC, Lucknow, with the chief complaint of a slowly progressing, painless swelling over the left side of lower jaw for the last 2 years. On palpation the swelling was non tender and hard with areas of firmness. The swelling extended from the superior to the inferior border, and from the angle of mandible to the midline. It expanded the mandible both buccally and lingually in the affected region (Figs. 1, 2). The mouth opening was normal, but the labial sulcus was obliterated (Fig. 3). There was no cervical lymphadenopathy and the findings on general examination were not significant. Orthopantogram (OPG) revealed multilocular radiolucency which extended from the left subcondylar region to the right first premolar region (Fig. 4). There was no displacement or resorption of any tooth in the mandible. Occlusal radiograph showed multilocular, honeycomb appearance with expansion of both the buccal and lingual cortical plates (Fig. 5). Computerized tomograph (CT) showed a well defined multilocular, expansile, lytic lesion which involved the body and ramus of mandible on the left side perforating the buccal and lingual cortical plates in the body of mandible, crossing the midline and extending till the right first premolar (Fig. 6).

Aspiration cytology (performed with $18 \mathrm{G}$ needle) was negative; hence a differential diagnosis of ameloblastic fibroma or odontogenic myxoma was made. An incisional biopsy was done under local anaesthesia in the affected 


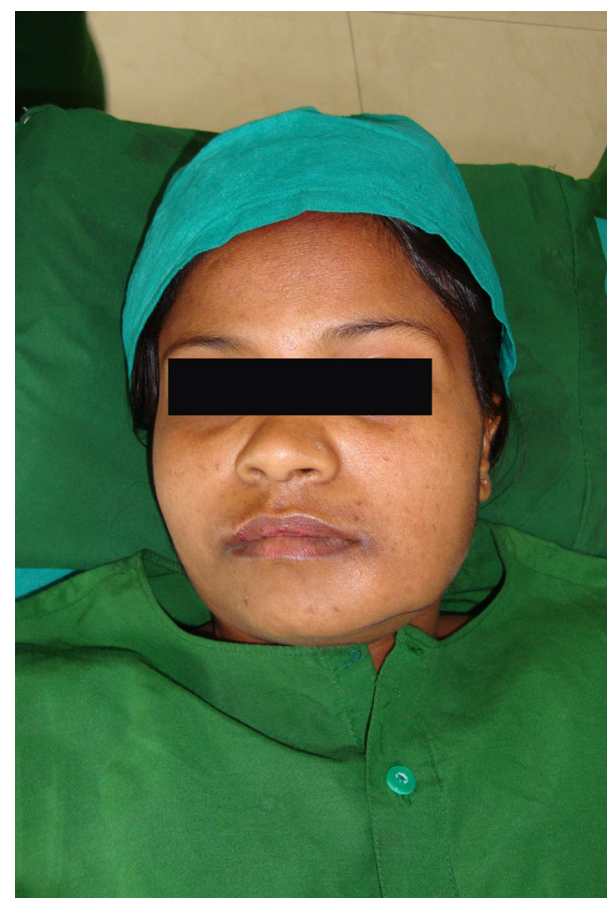

Fig. 1 Preoperative extraoral view from front

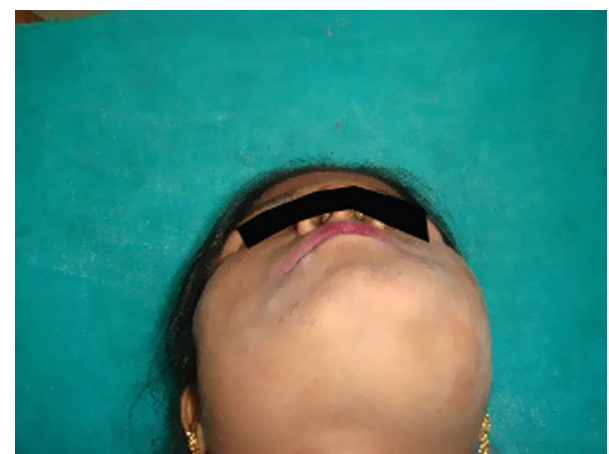

Fig. 2 Preoperative showing expansion of lower border and buccal aspect of mandible

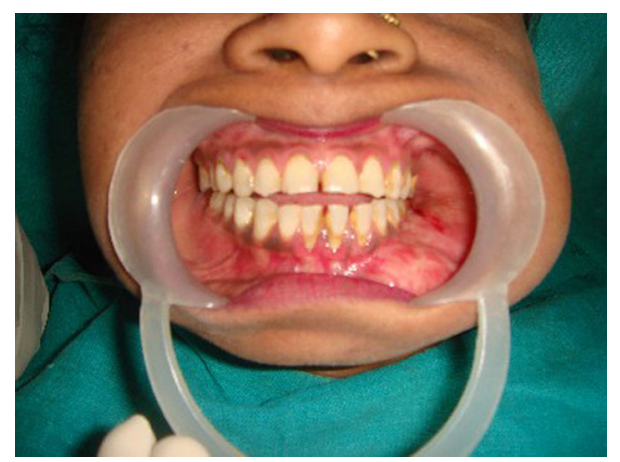

Fig. 3 Preoperative intraoral view showing expansion and obliteration of buccal vestibule

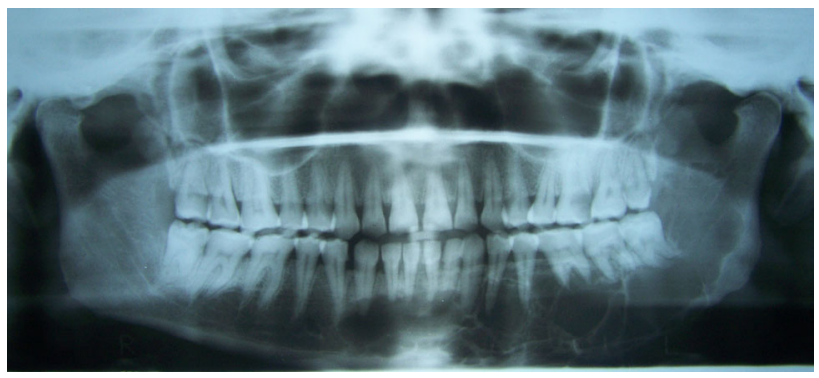

Fig. 4 Preoperative OPG depicting extensive lesion extending from left subcondylar region to right first premolar region

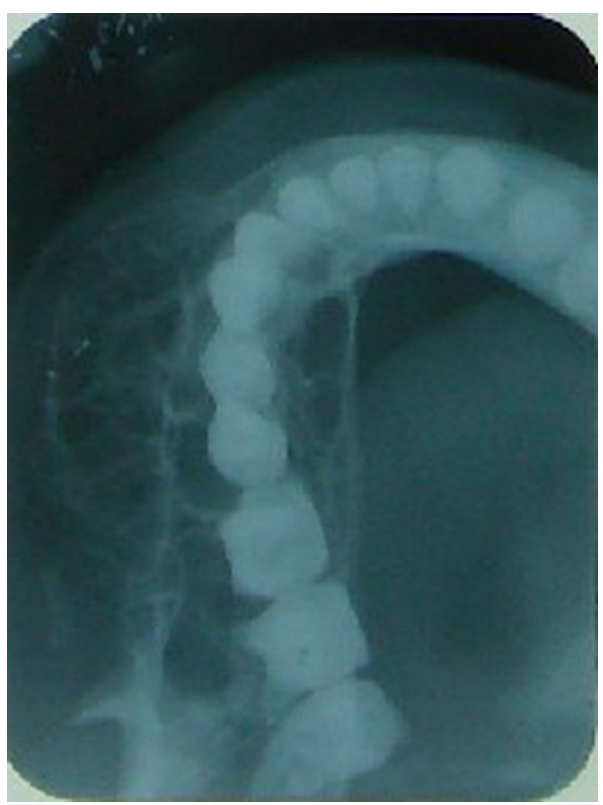

Fig. 5 Preoperative occlusal view of mandible showing multilocular honeycomb appearance

region by reflecting a two sided flap. The biopsy specimen was gelatinous in nature contained mucinous fluid which suggested the differential diagnosis of a myxoma. Histopathological examination of the specimen revealed proliferation of stellate to spindle shaped cells exhibiting ovoid, spindle or angular nuclei within a loose myxoid connective tissue stroma made of delicate collagen fibres and showed numerous engorged capillary sized vessels, which confirmed the diagnosis of odontogenic myxoma (Fig. 7).

Surgical resection of mandible was planned. After nasal intubation and surgical preparation, submandibular incision was given from left retromandibular region to right angle region and mandible was exposed by layer wise dissection (Fig. 8). First premolar was extracted and line of resection was marked with a flat fissure bur. Resection was done by gigli's saw and disarticulation was completed on the left side. No lip split was done in this case instead a mucosal incision was given from right to the left molar region, as an esthetic consideration. After resection Carnoy's solution 


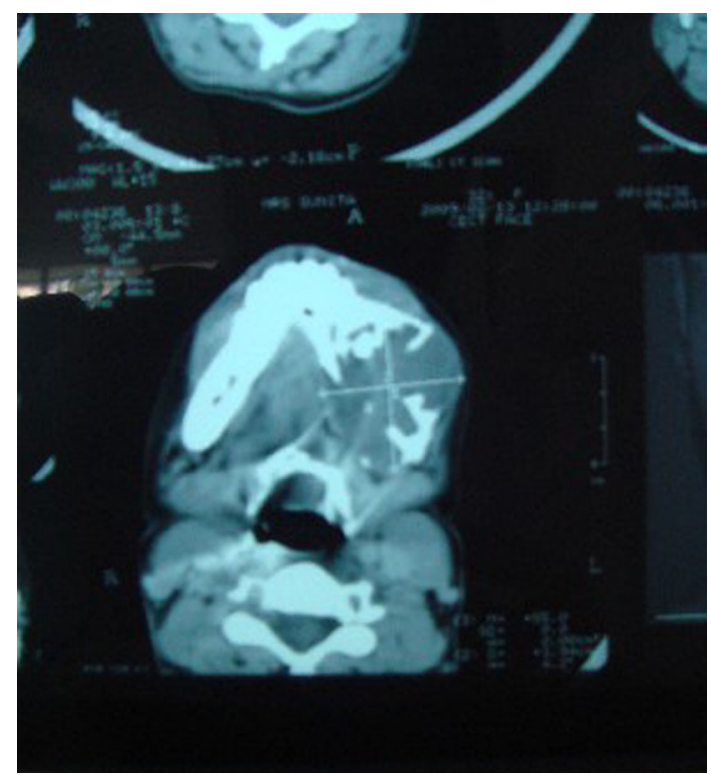

Fig. 6 Preoperative axial CT view showing expansion with destruction of buccal and lingual cortical plate

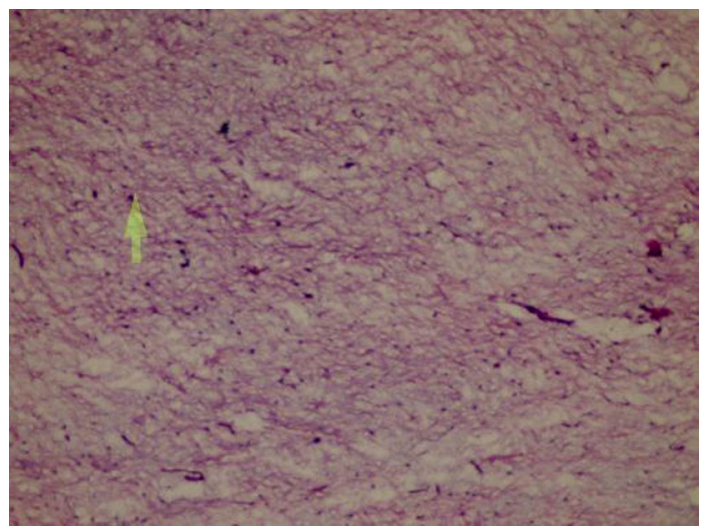

Fig. 7 Photomicrograph showing myxomatous tumour mass (H\&E, $4 \times)$

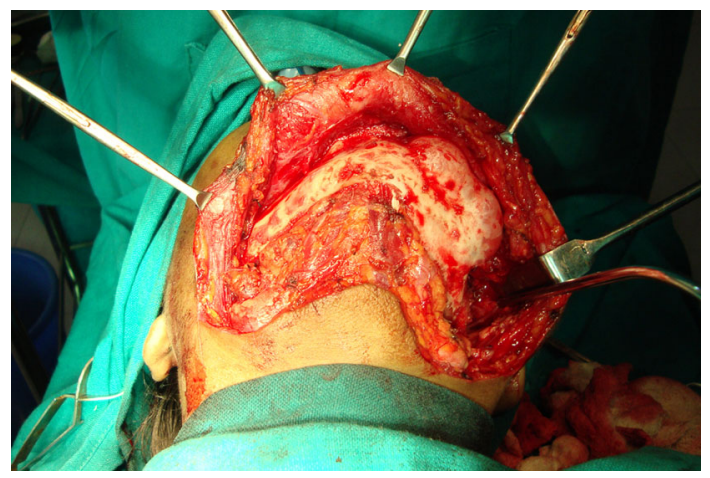

Fig. 8 Tumour exposure by submandibular incision
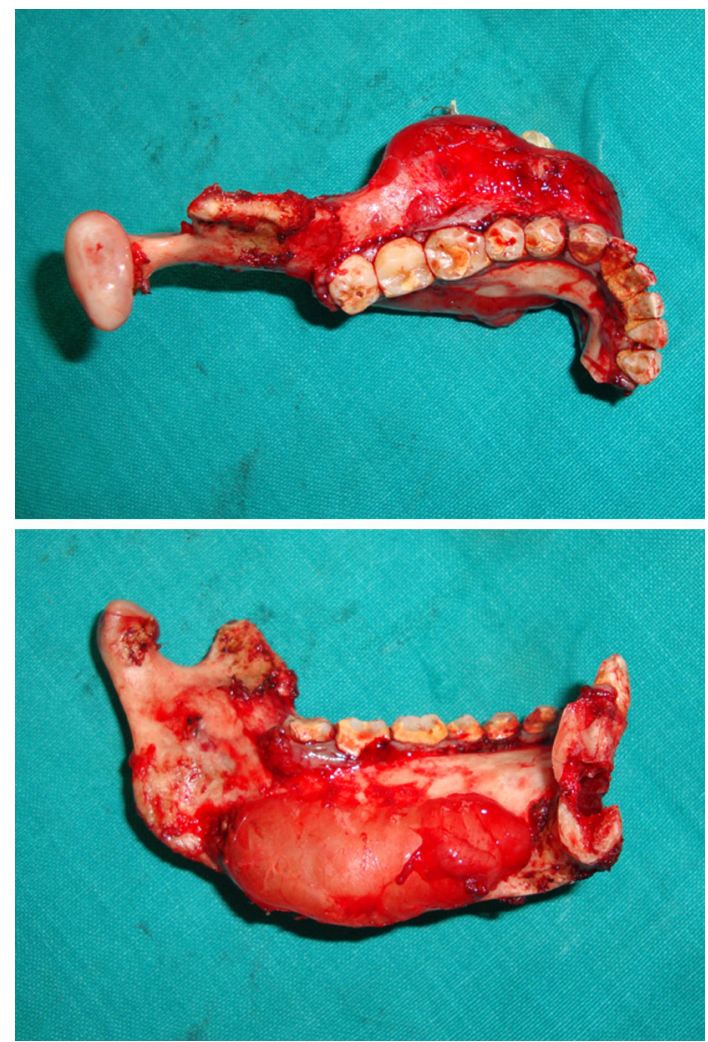

Fig. 9 Resected specimen showing expansion buccally and lingually

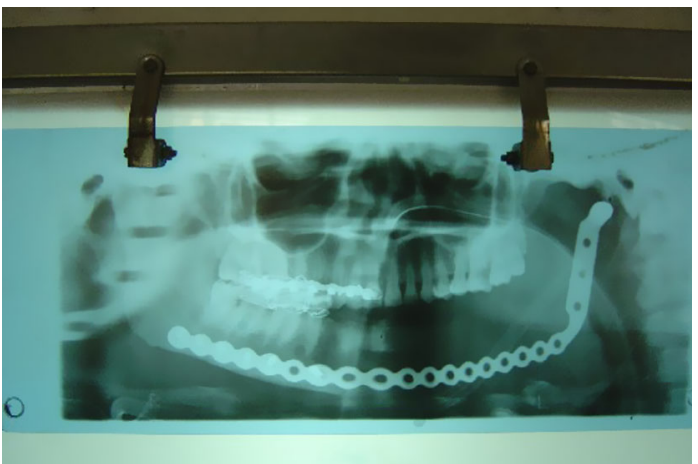

Fig. 10 Postoperative OPG

was applied to the cut margins of the remaining bone so as to destroy microscopic infiltration of tumor cells were present in the surrounding bone.

Intermaxillary fixation was achieved with Erich arch bar and wires and a condylar head reconstruction plate was fixed with six bicortical screws. The wound was closed in anatomical planes; extra orally by vicryl and silk and intraorally by vicryl only.

Postoperatively the patient was assessed with an OPG and Posteroanterior (PA) view of skull to confirm the position of head of condyle of condylar head plate in the glenoid fossa and contour of reconstruction plate with 


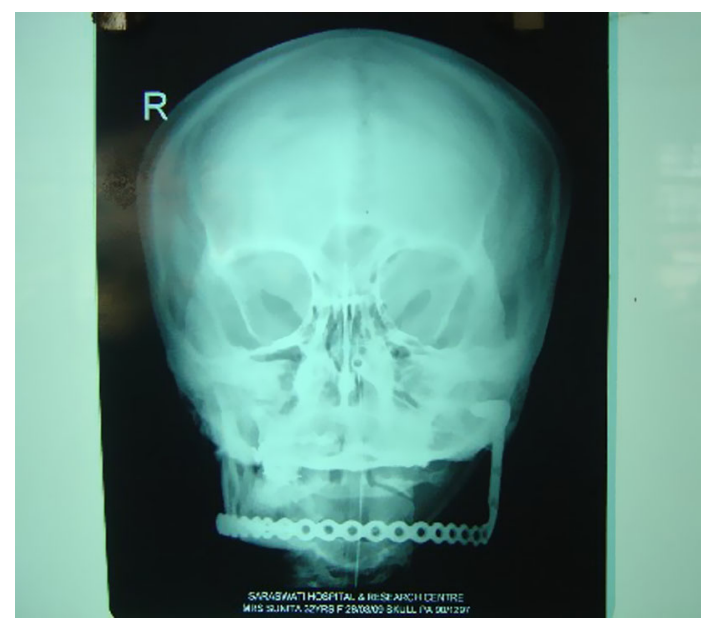

Fig. 11 Postoperative PA view of skull

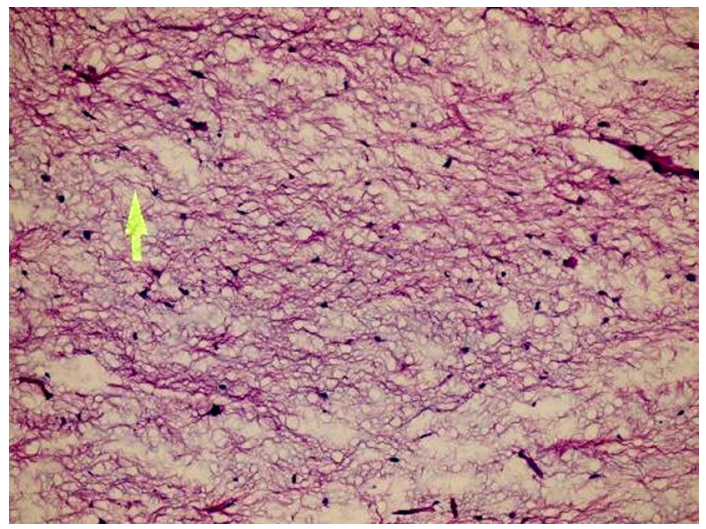

Fig. 12 Odontogenic myxoma histopathology (H \& E 10×)

respect to normal side. Head of condyle of reconstruction plate was well placed within glenoid fossa (Fig. 9) and contour of reconstruction plate was excellent (Fig. 10). The postoperative recovery was uneventful. Figure 11 showing the frontal view of the patient 1 month post operatively.

On gross examination the resected specimen was white in colour, with smooth gelatinous mass filling the expanded mandible (Fig. 12). Histopathological examination of lesion in this segment showed hypovascular, hypocellular lesion with abundance of myxoid component and characteristic stellate cells typical of odontogenic myxoma (Figs. 7, 13).

\section{Discussion}

Kaffe et al. [6] reported 7\% occurrence in first decade of life while reviewing 164 cases of odontogenic myxoma while Keszler et al. [7] reported $8.4 \%$ occurrence in less than 16 years of age while reviewing 367 cases of

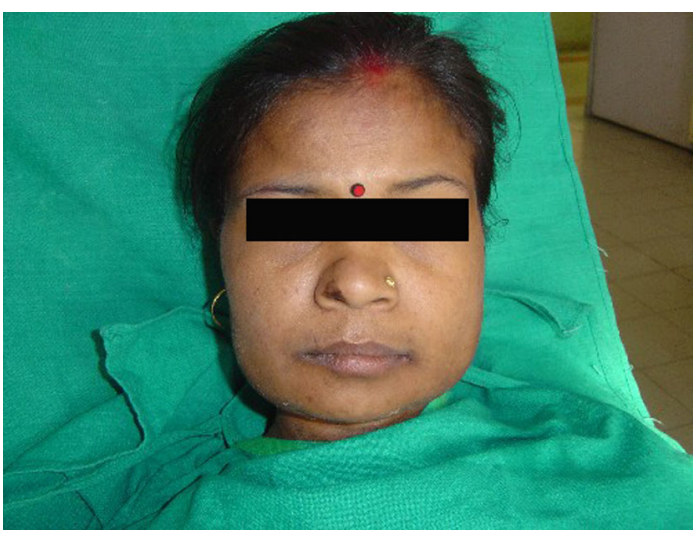

Fig. 13 One month postoperative frontal view

odontogenic myxoma. Stout defined myxoma as, "a true neoplasm made up of tissues resembling primitive mesenchyme". Thus, it is composed of stellate cells arranged in loose mucoid stroma which also contains delicate reticulin fibres. The lesion is benign and occurs commonly in the skin, the genitourinary tract, the gastrointestinal tract or in organs such as the liver, the spleen or even the parotid gland [10].

It is a rare tumour of the jaws, which arises from mesenchymal portion of tooth germ, the dental papilla, the follicle or the periodontal ligament. Evidence to suggest that this is an odontogenic neoplasm is based on the fact that the distribution of this tumour is primarily throughout the jaws and facial skeleton. Also, it is histologically similar to the stellate reticulum of developing tooth. The tumour is slow growing but is locally aggressive and has high recurrence rate [11]. On the basis of its clinical presentation it should be differentiated from dentigerous cyst, odontogenic keratocyst, ameloblastoma, central giant cell tumor, fibro osseous lesions, central hemangioma and various other odontogenic tumors which cause expansion of bone.

The odontogenic myxoma has hypocellular matrix and mucoid ground substance of glycosaminoglycans and chondroitin sulphate which accounts for its rapid growth and recurrence if inadequately removed. The recurrence is due to the local invasion into cancellous bone beyond radiographically visible margins, absent encapsulation unlike benign neoplasm and presence of mucoid ground substance [5]. Radiographically, it may produce several patterns: unicystic, multilocular, pericoronal (less often) and radiolucent-radiopaque (rare). When it occurs pericoronally with an impacted tooth, it is most likely to have a cyst like unilocular outline. Fine intralesional trabeculation occurs in most of the multilocular examples, as well as some of unicystic types, as a soap bubble, honey comb, or tennis-racket pattern. More than $50 \%$ of cases are multilocular and little less than $50 \%$ are unilocular. The 
unilocular variety tends to be small and is mostly located in the anterior region and the larger lesions are more likely to be multilocular and located in the posterior region. Margins may be poorly or well defined and sometimes border sclerosis may be present. The tumor may be scalloped between the roots of teeth. The odontogenic myxoma expands the cortical plates, showing as a smooth enlargement of the alveolus and basal bone. Loosening, displacement or resorption of teeth is occasionally seen. Sometimes, it perforates the cortical plates and produces a bosselated surface. The expanding tumor is soft on palpation when the cortical plates are destroyed and gives an impression of fluctance. Aspiration is non productive. Radiographically it should be differentiated from: odontogenic keratocyst, ameloblastoma, ameloblastic fibroma, central giant cell granuloma, central hemangioma, early stage of calcifying epithelial odontogenic tumor, early stage of calcifying odontogenic cyst and cherubism [12-14].

Macroscopically, it presents as a soft, glistening, gelatinous, and non encapsulated lesion. Microscopically, it is relatively hypocellular and composed of haphazardly arranged stellate, spindle-shaped, and round cells in an abundant loose myxoid stroma that contains only a few collagen fibrils [4, 15].On the basis of histopathology it should be differentiated from chondromyxoid fibroma and myxoid neurofibroma. Chondromyxoid fibroma is a well circumscribed tumor consisting of nodular myxoid tissue with scattered giant cells and areas of cartilagenous differentiation. It is located most commonly in the metaphyseal region of the long bones and rarely involves the jaws whereas myxoid neurofibroma tend to have scattered lesional cells that are positive for antibodies directed against S-100 protien. Even a enlarged dental follicle or a dental papilla of a developing tooth with myxoid change may be microscopically similar to a myxoma.

Various modalities have been suggested for treatment of myxoma. Small myxomas are generally treated by curettage, but careful follow up is necessary for at least 5 years. Larger lesions need extensive resection as myxomas are non encapsulated and tend to infiltrate the surrounding bone. Complete removal of a large tumour by curettage is often difficult to accomplish, and lesions of posterior maxilla, in particular should be treated more aggressively. Typical treatment of myxoma consists of surgical resection. The overall recurrence rate is approximately $25 \%$ and usually occurs during the first 2 years after removal. The advocated treatment of myxoma is surgical resection with a minimum of $1 \mathrm{~cm}$ of bone margin and one tumour free anatomic barrier because of its high recurrence rate [1]. Rotenberg et al. [16] suggested conservative or narrow resection of margins for myxomas of pediatric maxilla and found it to be effective. King et al. [5] did enucleation with peripheral osteotomy of $0.5-1 \mathrm{~mm}$ of bone and application of liquid nitrogen in two cases and found no recurrence. Liquid nitrogen cryosurgery may limit recurrence because of its ability to devitalize the organic content while leaving the inorganic framework intact. In this case resection of the affected mandible and application of Carnoy's solution on the edge of remaining bone was carried out.

\section{Conclusion}

Myxomas are very rare and locally aggressive tumors. They have a tendency to recur if not treated aggressively. An accurate histopathological diagnosis, sound surgical planning and its implementation are required to improve the prognosis.

\section{References}

1. Marx RE, Stern D (2003) Oral and maxillofacial pathology: a rationale for diagnosis and treatment. Quintessence Publishing, Hanover Park, II

2. Regezi JA, Keur DA, Courtney RM (1978) Odontogenic tumors: analysis of 706 cases. J Oral Surg 36(10):771-778

3. Daley TD, Wysocki GP, Pringle CA (1994) Relative incidence of odontogenic tumors and jaw cysts in Canadian population. Oral Surg Oral Med Oral Patho 77(3):276-280

4. Nevelle BW, Damm DD, Allen CM, Bouquet JE (2007) Oral and maxillofacial pathology, 2nd edn. Saunders, Philadelphia, pp 635-637

5. King TJ, Lewis J, Orvidas L, Kademani D (2008) Pediatric maxillary odontogenic myxoma: a report of 2 cases and review of management. J Oral Maxillofac Surg 66(5):1057-1062

6. Kaffe I, Naorti H, Buchner A (1997) Clinical and radiological features of odontogenic myxoma of jaws. Dentomaxillofac Radio 29:299

7. Keszler A, Dominquez FV, Giannunzio G (1995) Myxoma in childhood: an analysis of 10 cases. J Maxillofacial Surg 53:518

8. Slootweg PJ, Wittkampf AR (1986) Myxoma of the jaws: an analysis of 15 cases. J Maxillofac Surg 14:46

9. Barros RE, Dominquez FV, Cabrini RL (1969) Myxomas of the jaws. Oral Surg Oral Med Oral Pathol 27:225

10. Shafer WG, Hine MK, Levy BM (1999) A textbook of oral pathology, 4th edn. WB Saunders Company, Philadelphia, pp 160-161

11. Bucci E et al (1991) Odontogenic myxoma: report of a case with peculiar features. J Oral Maxillofac Surg 49(1):91-94

12. Booth PW, Schendel SA, Hausamen JE (1999) Maxillofacial Surgery, vol I, 2nd edn. Churchill Livingstone, New York, pp 502-503

13. Wood NK, Goaz NK (1998) Differential diagnosis of oral and maxillofacial lesions, 5th edn. Mosby, St. Louis, pp 342-343

14. Goaz PW, White SC (1997) Oral radiology. Principles and interpretation, 3rd edn. Mosby, St. Louis, pp 450-456

15. Muziol Lo et al (1996) Odontogenic myxoma of the jaws: a clinical, radiologic, immunohistochemical and ultrastructural study. Oral surg oral med Oral Pathol Oral Radiol 82:426

16. Rotenberg BW et al (2004) Myxomatous lesions of maxilla in children: a case series, review of management. Int $\mathrm{J}$ Pediatr Otorhinolaryngol 68:1251 